\title{
Neutron Scattering and Polymer Dynamics
}

\author{
Arantxa Arbe and Juan Colmenero* \\ Centro de Física de Materiales (CSIC/UPV-EHU) \\ Paseo Manuel de Lardizabal, 5 \\ 20018 San Sebastián (Spain) \\ *Also at Donostia International Physics Center and Materials Physics Center \\ (MPC) in San Sebastián (Spain)
}

\section{Introduction}

Nowadays polymers are ubiquitous in our daily life because they are durable, cheap to produce, easy to process and exhibit very versatile and favorable mechanical properties. For example, depending on temperature or time the same polymer may be viscous, rubber elastic, very tough with high impact strength or even brittle. Polymers are composed of macromolecules which are built up by a large number monomer units linked together by covalent bonds. Due to this connectivity, the relevant processes driving the dynamics in polymers depend on the length scale of observation. While at typical inter- and intra-macromolecular length scales $(\approx 10 \AA$ and below) polymer dynamics display the universal features of glass-forming systems, at larger distances the macromolecular character of the polymer chains prevails --there, entropy and topological constraints ('entanglements') play the major role. Carbon and Hydrogen are in most cases the main constituents of polymers. Therefore, neutron scattering (NS) combined with isotopic substitution (H/D labeling) is an extremely well suited technique to study the dynamical processes of polymeric materials at a molecular level. Motions of particular molecular groups or given polymer chains, e. g. in a polymer blend, can be selectively investigated by NS. However, the restricted dynamic window offered by this technique prevents a complete characterization of the complex polymer dynamics, including several multi-scale processes the characteristic times of which span over many orders of magnitude. The combination with other experimental techniques is, in most cases, essential to fully characterize the processes; in addition, the use of complementary MD-simulations has proved to be crucial for the interpretation of the NS results.

\section{Unraveling dynamical processes in polymers}

Among the different dynamical processes that take place in polymers, methyl group rotation is perhaps the simplest one, since all the relevant interactions on the methyl group can be condensed in an effective mean-field one-dimensional potential. However, the disorder present in any structural glass leads to a distribution of potential barriers for methyl group rotation. Only taking into account the existence of such a distribution, as in the Rotation Rate Distribution Model (RRDM) first proposed in 1994 [1], it is possible to interpret the neutron spectra associated to methyl group dynamics in glassy systems. In particular, quantum rotational tunneling of methyl groups in polymers was expected for a long time, but only quite recently (1998) was directly observed by high-resolution NS techniques [2]. Figure 1 shows the dramatic impact of disorder on the appearance of the tunneling lines in a typical glass-forming polymer: instead of inelastic lines, an apparent quasielastic broadening is detected. For this reason, the presence of tunneling signatures in the low-temperature spectra of glassy materials was overlooked for many years.

Neutron scattering has also shed light on the molecular origin of the so-called secondary relaxations detected by relaxation techniques on glassy polymers. The combination of dielectric 
and mechanical spectroscopy and NS has allowed identifying the molecular motions behind the $\gamma$ relaxation in engineering thermoplastics (relevant for their use as commodities) like the wellknown polycarbonate [3]. Furthermore, a pioneering neutron spin echo (NSE) investigation of polybutadiene [4] addressed the basic question of the origin of the Johari-Goldstein relaxation and its relationship with the structural relaxation. The neutron results suggested statistically independence of both processes, establishing a new method for analyzing dielectric data of polymeric materials.

Since its freezing leads to the glassy state, the structural or $\alpha$-relaxation is the most relevant dynamical process of glass-forming materials. Neutron scattering, and in particular NSE, offers the unique possibility of directly observing this process at a molecular level by following the evolution of the dynamic structure factor on deuterated samples at the first amorphous halo (inter-molecular peak). Thereby, the decay of the correlations between neighboring chain segments (structural units) due to the $\alpha$-relaxation can be assessed. In the last decades, this kind of studies have been extended to a large number of polymers and other glass-forming systems [5], revealing the main features of the structural relaxation: universality, scaling and stretching. In addition, NS on protonated samples has allowed characterizing the atomic motions in the $\alpha$ relaxation-regime. Worth of remark is the correlation found between the non-exponential behavior and the momentum-transfer (Q) dependence of the intermediate scattering function [6], that qualifies these motions as Gaussian sublinear diffusive processes.

In the microscopic time window, two other processes are also ubiquitous in glass-forming systems: the Boson peak and the so-called fast dynamics. Fourier transformation into the time domain to obtain the intermediate scattering function (applied for the first time to polymers in [7]) facilitated the interpretation of the fast process as the relaxation mechanism uncoupled from the system. However, the origin of both, fast dynamics and Boson peak, is still subject of controversy.

All the above mentioned processes are relevant at intra- and/or inter-molecular length scales, and characteristic for the glass-forming nature of polymeric materials. Increasing the length-scale of observation, a crossover toward entropy-driven chain dynamics occurs. NSE experiments on the single-chain dynamic structure factor of unentangled polymers have proven the validity of the so-called Rouse model at length-scales where the particular microscopic (chemical) details of the monomers become irrelevant [5]. However, despite the recent experimental efforts made to characterize polymer motions in the intermediate-length scale region, understanding the crossover from segmental to Rouse dynamics demands for further theoretical developments [5]. This is still quite a white field in polymer physics.

\section{Synergy with Molecular Dynamics simulations}

Since NS explores a limited region of the reciprocal space, magnitudes like atomic mean squared displacements are not easily accessible (or even impossible to obtain). Therefore, a rational combination of NS experiments and molecular dynamics (MD) simulations is essential to fully characterize the dynamical processes in polymers. A successful methodology should involve a careful validation of the MD-simulations through direct comparison of the calculated magnitudes with experimental results. Once the reliability of the simulated cell is proved, the MDsimulations can be exploited to calculate magnitudes that cannot be accessed by NS. Sometimes, they also might even motivate state-of-the-art experiments [8]. Applying this kind of synergetic methodology the atomic motions in the $\alpha$-regime could be characterized in detail, revealing nonGaussian features in the region between the microscopic times and the sublinear regime. These effects manifest as deviations from the Q-dependence experimentally observed in previous NS experiments at low Qs [6] (see Fig. 2). Last not least, carefully validated cells allow thorough 
checks for theoretical frameworks like the Mode Coupling Theory (MCT) for the glass transition. The recent successful application of MCT phenomenological predictions to polymer systems (see [9] and references therein) suggests a higher-order MCT transition for real polymers arising from the simultaneous occurrence of two mechanisms leading to dynamic arrest: i) packing, of intermolecular character and present in all glass-forming systems; ii) barriers for conformational changes, of intramolecular origin, which are specific of macromolecular systems.

\section{Toward complex systems based on polymers}

In the field of polymers, the current trend --as in soft matter in general-- is to study systems of increasing complexity, which are more relevant for technological and biological applications. This is facilitated by the capability of NS to selectively probe spatially resolved dynamical modes. One clear achievement is the establishment at a molecular level of the dynamic heterogeneity in thermodynamically miscible polymer blends. As can be seen in Fig. 3, blending two homopolymers with different mobilities affects the dynamics of both kinds of chains; however, we can still clearly distinguish the dynamics of both components within the blend. Recently, it has been put forward the role played by the dynamic asymmetry between different components in polymer blends. This asymmetry can lead to confinement effects when the slow component is effectively frozen in the relevant timescale of the fast one [10]. We note that nowadays the investigation of confinement effects on polymer dynamics is a very active field. Is worth of remark the recent first experimental microscopic evidence of the dilution of the total entanglement density in a polymer melt under strong confinement [11]. This observation was possible thanks careful NSE measurements on extremely well defined samples (polymers confined in almost perfectly aligned cylindrical pores of nanometric diameter).

Interestingly enough, confinement effects can also emerge in relatively simple polymers like homopolymers with long alkyl side-groups. Recently the selectivity of neutron scattering combined with isotopic substitution has been exploited to study the structure and dynamics of poly(n-alkyl methacrylates) [12]. Diffraction nicely demonstrates the nanosegregation of mainchains and side-groups (see Fig. 4). Moreover, NSE has allowed separately following the structural relaxation of both subsystems. Contrary to the standard behaviour found at the mainchain level, the correlations involving long side groups within the alkyl nanodomains relax through an exotic logarithmic decay --probably a signature of nanoconfinement of the sidegroups by the more rigid main chains.

\section{Conclusions}

Neutron scattering has proven to be a crucial technique to unravel polymer dynamics. Its combination with other experimental techniques and MD-simulations during the last decades has allowed deciphering most of the diverse and complex dynamical processes taking place in homopolymers. We are confident that this methodology is also the right tool to face the challenge of unveiling polymer dynamics at different length scales in more complex systems, as demanded by the current trends in the soft-matter field.

\section{Acknowledgments}

We are indebted to our research group in San Sebastián, in particular to Prof. A. Alegría, and Drs. A. J. Moreno and S. Arrese-Igor. We also thank the group of Prof. D. Richter in Jülich and our colleagues at the different neutron facilities, in particular Drs. B. Frick and B. Farago at the ILL, for many years of fruitful collaboration. 


\section{References}

[1] J. Colmenero, A. J. Moreno, and A. Alegría, Progress in Polymer Science 30, 1147 (2005) and references therein

[2] J. Colmenero, R. Mukhopadhyay, A. Alegría, B. Frick, Phys. Rev. Lett. 80, 2350 (1998)

[3] S. Arrese-Igor, O. Mitxelena, A. Arbe, A. Alegría, J. Colmenero and B. Frick, Physical Review E 78, 021801 (2008) and references therein

[4] A. Arbe, U. Buchenau, L. Willner, D. Richter, B. Farago, J. Colmenero, Phys. Rev. Lett. 76, 1872 (1996)

[5] D. Richter, M. Monkenbusch, A. Arbe and J. Colmenero, Advances in Polymer Science, Vol. 174 (2005) and references therein

[6] J. Colmenero, A. Alegría, A. Arbe, B. Frick, Phys. Rev. Lett. 69, 478 (1992)

[7] J. Colmenero, A. Arbe, A. Alegría, Phys. Rev. Lett. 71, 2603 (1993)

[8] A. Arbe, J. Colmenero, F. Alvarez, M. Monkenbusch, D. Richter, B. Farago, B. Frick, Phys. Rev. Lett. 89, 245701 (2002)

[9] S. Capponi, A. Arbe, F. Alvarez, J. Colmenero, B. Frick and J. P. Embs, Journal of Chemical Physics 131, 204901 (2009)

[10] J. Colmenero and A. Arbe, Soft Matter 3, 1474 (2007) and references therein

[11] J. Martín, M. Krutyeva, M. Monkenbusch, A. Arbe, J. Allgaier, A. Radulescu, P. Falus, C. Mijangos, J. Colmenero and D. Richter, Phys. Rev. Lett. (submitted)

[12] A. Arbe, A.-C. Genix, J. Colmenero, D. Richter and P. Fouquet, Soft Matter 4, 1792 (2008) 


\section{FIGURES}

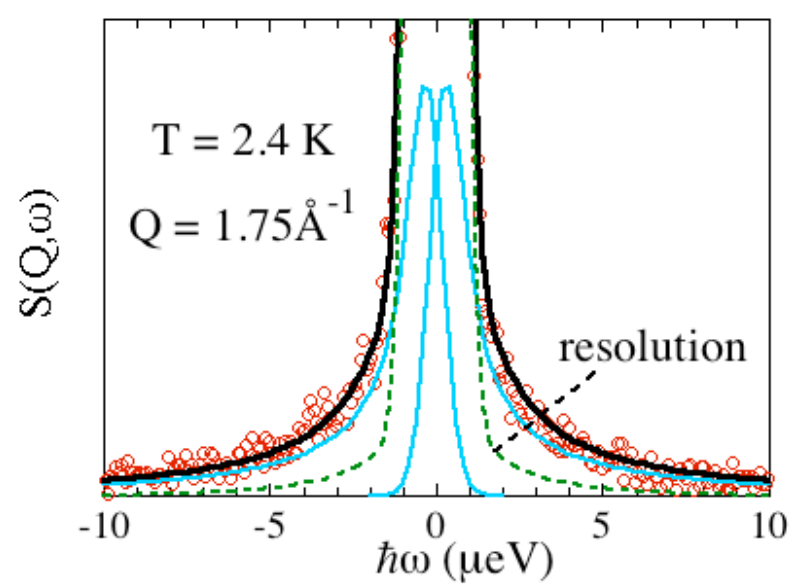

Figure 1. Spectra of poly(vinyl acetate) showing tunneling effects as apparent quasielastic broadening. Thick solid line: description in terms of the RRDM. Thin solid lines: inelastic contribution of the rotational tunneling processes (from [2]).

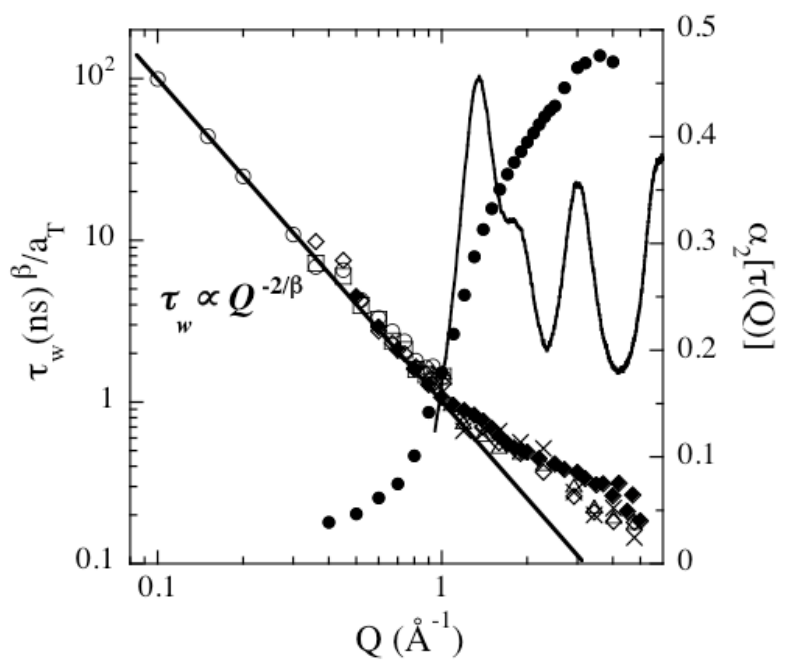

Figure 2. Q-dependence of the characteristic time of the $\alpha$-relaxation in polyisoprene (empty symbols: NS; full symbols: MD-simulations). Results corresponding to different temperatures have been superimposed and then exponentiated to the power of $\beta$ (stretching parameter). Solid line through points: Gaussian Q-dependence. Non-Gaussian parameter at $\tau_{\mathrm{w}}(\mathrm{Q})$ (filled circles) and S(Q) (both from simulations) are also included (from [8]). 




Figure 3: Normalized backscattering results at $330 \mathrm{~K}$ and $\mathrm{Q}=1.1 \mathrm{~A}^{-1}$. From above: PI (polyisoprene), PI in PI/PVE (poly(vinylethylene)), PVE in PI/PVE, PVE, and instrumental resolution. Blend concentration: $50 \% / 50 \%$ [10].

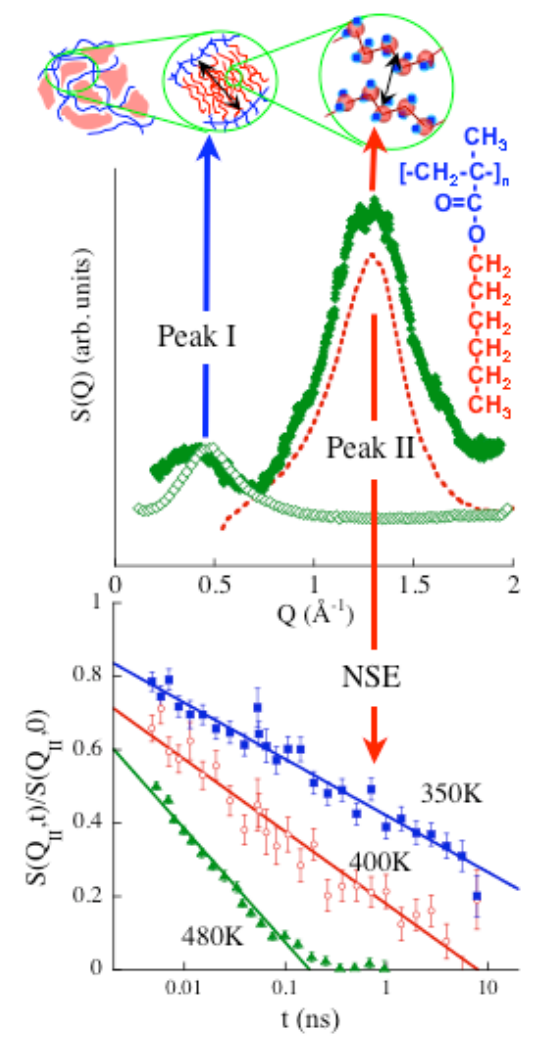

Figure 4: Neutron diffraction pattern of fully deuterated PHMA (poly(hexyl methacrylate)) (full symbols) and PHMA deuterated only along the main-chain (empty diamonds). Dotted line: $S(Q)$ of bulk polyethylene. Cartoon: interpretation of the correlations giving rise to the peaks in $S(Q)$. The dynamic structure factor at Peak II is shown in the lower part. Solid lines: fits to a logarithmic expansion. 